\title{
Toxoplasma gondii Infection and Related Risk Factors among Blood Donors in Northwest Iran
}

\author{
Reza Bahhaj,, ${ }^{1,2}$ Ehsan Ahmadpour,, ${ }^{3,4}$ Mahmoud Mahami-Oskouei, ${ }^{1,2,}$ Esmaeil Fallah,, ${ }^{2}$ Karim \\ Shamsasenjan, ${ }^{5,6}$ and Abdolrasoul Safaiyan ${ }^{7}$ \\ ${ }^{1}$ Immunology Research Center, Tabriz University of Medical Sciences, Tabriz, Iran \\ ${ }^{2}$ Department of Parasitology and Mycology, Faculty of Medicine, Tabriz University of Medical Sciences, Tabriz, Iran \\ ${ }^{3}$ Infectious and Tropical Diseases Research Center, Tabriz University of Medical Sciences, Tabriz, Iran \\ ${ }^{4}$ Toxoplasmosis Research Center, Mazandaran University of Medical Sciences, Sari, Iran \\ ${ }^{5}$ Hematology and Oncology Research Center, Tabriz University of Medical Sciences, Tabriz, Iran \\ ${ }^{6}$ Blood Transfusion Research Center, High Institute for Research and Education in Transfusion Medicine, Tehran, Iran \\ ${ }^{7}$ Department of Biostatistics and Epidemiology, Faculty of Health, Tabriz University of Medical Sciences, Tabriz, Iran \\ "Corresponding author: Mahmoud Mahami-Oskouei, Immunology Research Center and Department of Parasitology and Mycology, Faculty of Medicine, Tabriz University of \\ Medical Sciences, Tabriz, Iran. Tel/Fax: +98- 4133373745, E-mail: mahamim@tbzmed.ac.ir
}

Received 2016 October 08; Revised 2017 February 19; Accepted 2017 March 04.

\begin{abstract}
Background: Toxoplasmosis, a worldwide parasitic infection, is considered as a transfusion-transmitted disease.

Objectives: Due to ignorance about Toxoplasma infection in blood donors monitoring and its important role in provision of safe blood and blood products and also poor information about the seroprevalence of $T$. gondii infection in blood donors, this study was conducted to assess anti-Toxoplasma gondii antibodies and associated risk factors among blood donors in northwest of Iran.

Methods: In this cross- sectional study, 194 serum samples were collected from healthy blood donors who referred to Tabriz Blood transfusion organization (East Azerbaijan, Iran) during April and June 2014 and tested for anti-T. gondii IgM and IgG antibodies using chemiluminescence immunoassay. The questionnaire consisting of demographic and risk factors were filled for all the participants. Results: From 194 donors, 75 (38.66\%) and 2 (1.03\%) individuals were seropositive for anti-T. gondii IgG and IgM antibodies, respectively. In the present study, anti-T. gondii antibodies in first-time donors was significantly higher than those in regular blood donors $(\mathrm{P}<0.05)$. A significant correlation was observed between T. gondii infection and age $(\mathrm{P}<0.05)$; however, there were no relationship between Toxoplasma infection and other factors.

Conclusions: Considering the medical importance of blood transfusion and high rate of anti- $T$. gondii antibodies in healthy blood donors, it seems that effective monitoring of infection is required to prevent transfusion- transmitted toxoplasmosis.
\end{abstract}

Keywords: Chemiluminescence, Blood Transfusion, Blood Donor, Iran, Toxoplasma gondii

\section{Background}

Toxoplasma gondii is an intracellular protozoan parasite that causes toxoplasmosis. This parasite is an opportunistic pathogen that infects all warm blood animals including humans (1). It has been estimated that up to one third of the world's population are infected by T. gondii. Despite the worldwide distribution and prevalence of $T$. gondii infection, it is usually mild and self-limiting in immunocompetent hosts (2-6). However, toxoplasmosis can cause severe and fatal disease in immunocompromised patients and congenitally infected fetuses and newborns (2, 7-9). It has been proven that there are 3 principle routes for Toxoplasma transmission: (1) the consumption of oocysts contaminated water or vegetables, (2) ingestion of raw/undercooked meat containing tissue cysts, and (3) congenital transmission $(1,10)$. Furthermore, toxoplasmosis is considered as one of the transfusion-transmissible diseases and in rare cases can be transmitted through an organ transplant or blood transfusion. T. gondii is capable to invade and replicate in all nucleated cells of the body and survive for several weeks in stored blood (11-14). Today, there has been a significant reduction in the number of transfusion- transmitted infections; however, screening for T. gondii in blood and blood product is not obligatory, and subsequently blood borne toxoplasmosis remain a concern $(1,9,11)$. Considering the serious consequences of toxoplasmosis in immunocompromised individuals (such as patients with acquired immunodeficiency syndrome and transplant recipients) and neglecting the T. gondii infection monitoring in blood donors, it is of paramount importance to have knowledge about the T. gondii prevalence in blood donor population for provision of safe blood and blood products (2, 9-11). Besides, to date, there has been no recommended guideline or procedure for the prevention 
of transfusion- transmitted toxoplasmosis.

\section{Objectives}

Considering the poor information on the seroprevalence of T. gondii infection in blood donors, this crosssectional study was conducted to assess IgM and IgG antiT. gondii antibodies and related risk factors among blood donors in northwest Iran.

\section{Methods}

\subsection{Study Design and Sample Collection}

We performed a cross-sectional study in Tabriz blood transfusion organization (TBTO) in East Azerbaijan province, northwest of Iran (Figure 1). In this survey, we collected 194 sera samples (188 males and 6 females; 18 - 60 years old) from healthy blood donors who referred to TBTO during April and June 2014. To estimate the sample size, we considered $95 \%$ confidence interval with a standard deviation of 1.89 and 0.55 maximum error. Before blood donation, each participant completed a questionnaire about demographic data and risk factors including gender, age, education, times of blood donation, contact with cats, and eating raw animal products. All blood samples were serologically screened for human immunodeficiency virus (HIV), hepatitis C virus (HCV), hepatitis B virus surface antigen (HBsAg), and Treponema pallidum according to TBTO policy. Healthy blood donors referred to blood transfusion organization were enrolled in this study, and the samples that were positive for human immunodeficiency virus (HIV), hepatitis C virus (HCV), hepatitis B virus surface antigen (HBsAg), Treponema pallidum, and chylomicronic serums were excluded from the present investigation; thus, 16 serum samples were excluded according to these criteria. Then, 500 micro liters of serum samples from healthy blood donors that were seronegative for HIV, HCV, HBsAg, and T. pallidum were collected and transferred to the Department of Parasitology, Tabriz University of Medical Sciences and were kept at $-20^{\circ} \mathrm{C}$ until use.

\subsection{Ethical Consideration}

This study was approved by research council of Tabriz international University of Medical Sciences (Aras). (No. 93.1-3.3, May 2014). After obtaining written informed consent, we collected blood samples from all the participants.

\subsection{Chemiluminescence Immunoassay (CLIA)}

To evaluate the anti-T. gondii antibodies, we analyzed all the serum samples using chemiluminescence immunoassay (CLIA) with Diasorin Liaison calibrated analyzer (Germany) by a commercially available kit (Diasorin, Italy). Both tests (IgG and IgM) were performed in Tabriz Noor private laboratory following the instructions of the manufacturer. Values of more than 8.8 and 8 international units (IU)/mL were considered to be positive for IgG and IgM, respectively.

\subsection{Statistical Analysis}

Statistical analysis was performed by SPSS ver. 13 for windows; normality was evaluated by K-S test for quantitative variables. Chi-square and logistics tests were used to assess the relationship between positive and negative groups of IgG and IgM with some variables. One way ANOVA test was used to determine the relationship between quantitative IgG and IgM with some important variables, as the predictors and confounding variables were controlled. Error-bar diagrams were used to show antibody levels and number of blood donations in the 2 groups (less than 5, 5 and more). The level of significance was set at 0.05 , and all results were expressed as frequency (percent) and Mean \pm SD.

\section{Results}

A total of 194 blood donors were enrolled in this study. Most of the participants were males (96.9\%), with the mean age of 37.04 years. Of the 194 participants who were tested, 75 (38.66\%) and 2 (1.03\%) were seropositive for anti-T. gondii IgG and IgM antibodies, respectively. Demographic patterns and the anti-T. gondii seropositivity are summarized in Table 1. A significant correlation was found between IgG antibodies and age; however, no relationship was found between Toxoplasma infection and other factors.

The titer of antibodies gradually decreased according to the number of donations. Based on the results, anti$T$. gondii antibodies in first-time donors was significantly higher than in regular blood donors $(\mathrm{P}<0.05)$. Also, $T$. gondii seropositivity was associated with the donation status of the blood donor ( $<5$ or $\geq 5$ donation) (Figure 2$)$.

\section{Discussion}

Toxoplasmosis is one of the most prevalent parasitic infections in humans that infects approximately one-third of the world's population (1). With respect to T. gondii viability 


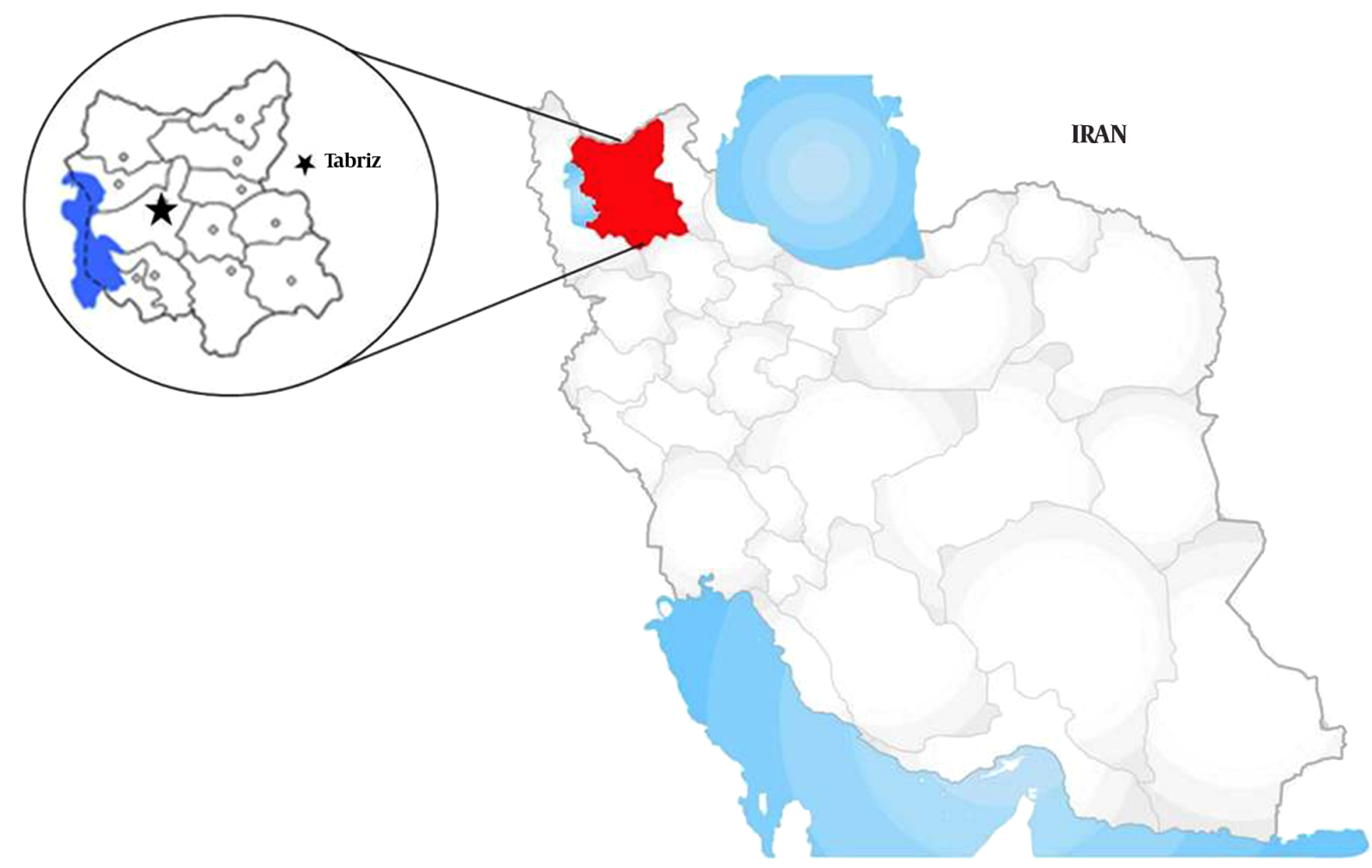

Figure 1. Geographical Location of the Study Area
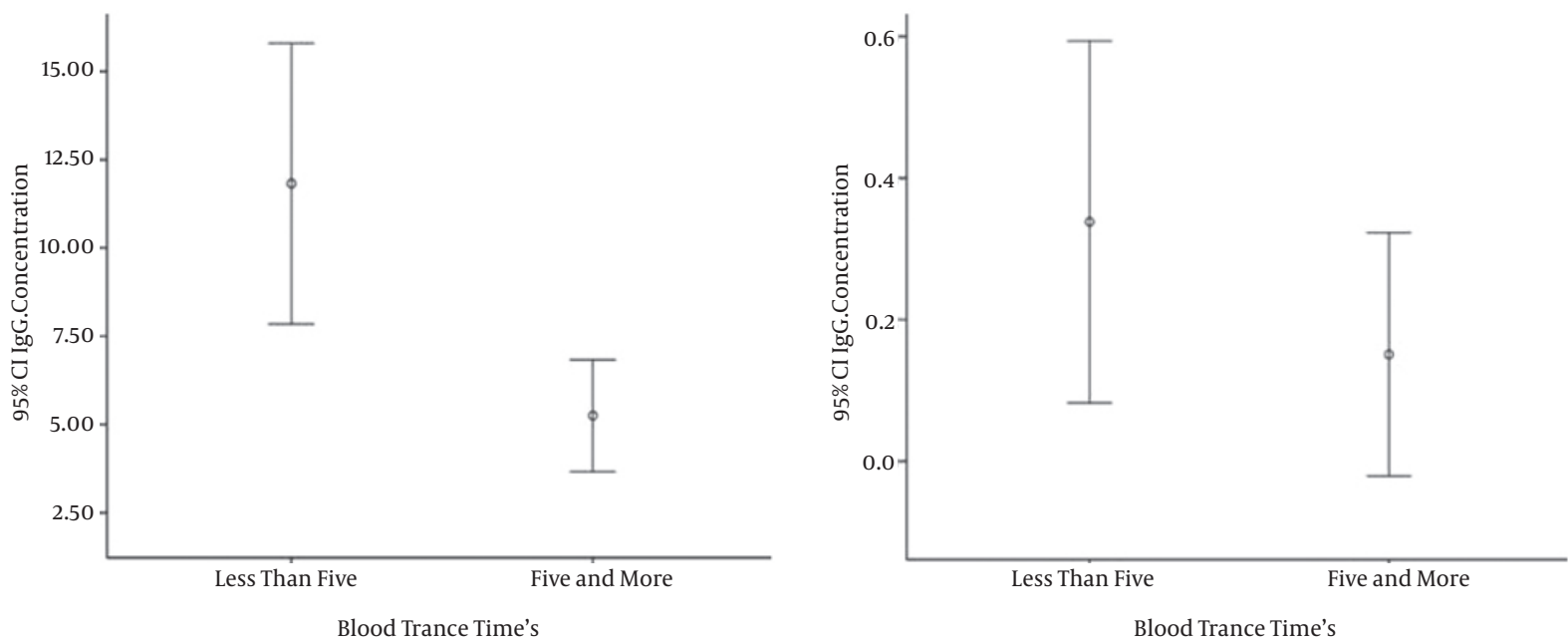

Figure 2. Relation Between IgG (left) and IgM (right) Antibody Levels and Times of Blood Donation

in blood products for several weeks, T. gondii can be transmitted through a blood transfusion. Therefore, vulnerable clinical groups such as immunocompromised patients are at a high risk for acute and severe toxoplasmosis by the donated blood $(2,9,11)$. Currently, no specific laboratory screening of blood donations is performed for T. gonii infection (11). In the present study, we found that 75/194 (38.66\%) and 2/194 (1.03\%) of healthy blood donors were seropositive 


\begin{tabular}{|c|c|c|c|c|c|}
\hline Variable & No. & IgG Positive & PValue & IgM Positive & PValue \\
\hline Gender & & & 0.251 & & 0.939 \\
\hline Male & 188 & $74(39.36)$ & & $2(1.06)$ & \\
\hline Female & 6 & $1(16.67)$ & & $0(0)$ & \\
\hline Age, $y$ & & & 0.001 & & - \\
\hline$<30$ & 42 & $8(19.4)$ & & $0(0)$ & \\
\hline $30-34$ & 46 & $12(26.08)$ & & $1(2.17)$ & \\
\hline $35-40$ & 35 & $15(42.85)$ & & $0(0)$ & \\
\hline$>40$ & 71 & $40(56.33)$ & & $1(1.4)$ & \\
\hline Education & & & 0.171 & & - \\
\hline School and below & 47 & $24(51.06)$ & & $2(4.26)$ & \\
\hline Diploma & 57 & $22(38.6)$ & & $0(0)$ & \\
\hline Undergraduate & 66 & $20(30.3)$ & & $0(0)$ & \\
\hline Postgraduate & 24 & $9(37.5)$ & & $0(0)$ & \\
\hline Contact with cat & & & 0.234 & & 0.909 \\
\hline Yes & 9 & $5(55.56)$ & & $0(0)$ & \\
\hline No & 185 & $70(37.84)$ & & $2(1.08)$ & \\
\hline Eating raw animal products & & & 0.129 & & 0.656 \\
\hline Yes & 80 & $36(45)$ & & $1(1.25)$ & \\
\hline No & 114 & $39(34.21)$ & & $1(0.88)$ & \\
\hline
\end{tabular}

${ }^{\mathrm{a}}$ Values are expressed as No. (\%).

for anti-T. gondii IgG and IgM antibodies, respectively. The presence of IgM antibodies occurred following acute infection, however, high level of IgM may last for a long time. The results of the current study revealed low seropositivity of IgM (acute infection) despite high the rate of anti.T. gondii IgG antibodies (latent infection). Results of the current investigation are comparable to those published from studies in Tehran and Kerman, Iran $(13,15)$. In addition, similar T. gonii seroprevalence studies on blood donors have been conducted in other countries such as Taiwan, India, and Mexico. The reported rates vary from $7.4 \%$ to $51.8 \%$ for IgG and $0.28 \%$ to $5 \%$ for IgM anti-T. gondii antibodies $(12,16-$ 18). These differences may be due to sample size, mean age of the participants, geography, and sensitivity of detection methods.

Diagnosis of $T$. gondii infection routinely depends on serological assays. Hence, tests with high sensitivity and specificity are required (19-23). Prusa et al. compared electrochemiluminescence assay with Sabin-Feldman dye test and ELISA as a reference test in gestational infection with T. gondii. They demonstrated that electrochemiluminescence is a useful method to detect $T$. gondii infection with high sensitivity and specificity of $97.1 \%$ and $100.0 \%$, respectively (24). Because of low specificity of ELISA, we analyzed the anti-T. gondii IgG and IgM antibodies using chemiluminescence immunoassay.

Our findings revealed that among the blood donors, age group was the only significant risk factor for $T$. gondii seropositivity. This indicates that seropositivity increases significantly with age and the peak of the infection rate is detected in the 40- year- old age group. The reason for the high rate in older age group is not clear, but it may be due to long time exposure by individuals during their life. In line with these results, Mahmoudvand et al. reported a similar relationship between $T$. gondii seroprevalence and age groups in southeast of Iran (15). No correlation was found between $T$. gondii infection and other mentioned demographic factors. Our results revealed that the males were more infected than the females, but the difference was not significantly different $(\mathrm{P}>0.05)$. Our study results, in agreement to study of Alvarado-Esquivel et al. in Mexico, did not show an association between gender and infection (18). However, it was in contrast with the findings of Elhence et al. in India (12). Our study limitation was the 
number of female blood donors, which was much lower than the male donors and lead to showing a low seropositivity rate in females; thus, it may not be a true reflection of toxoplasmosis in the society. In the present investigation, regular blood donors had lower antibody levels, which may be due to a decrease in the titer of antibodies following blood donations. It seems that further studies including a large number of blood donors are needed to better elucidate the potential risk factors associated with T. gondii infection.

\subsection{Conclusions}

Based on our findings, T. gondii infection is highly prevalent among healthy blood donors in northwest of Iran. In addition, we found a low IgM seropositivity despite the high rate of IgG. Although the seropositivity of acute infection (IgM) among blood donors was low in this study, it seems that the pretransfusion screening will be a helpful option for prevention of transfusion-transmitted toxoplasmosis in regions with high endemicity. Also, further studies are needed to focus on molecular epidemiology of $T$. gondii infection in blood donors and more attention should be paid to this neglected parasitic disease.

\section{Acknowledgments}

This article was derived from the master's thesis of the first author (No. 93.1-3.3).

\section{Footnotes}

Authors' Contribution: All authors contributed in study design, sample collection, analysis, and writing the manuscript.

Funding/Support: This study was financially supported by immunology research center, Tabriz University of Medical Sciences, Iran.

\section{References}

1. Hill DE, Chirukandoth S, Dubey JP. Biology and epidemiology of Toxoplasma gondii in man and animals. Anim Health Res Rev. 2005;6(1):4161. doi: 10.1079/AHR2005100. [PubMed: 16164008].

2. Ahmadpour E, Daryani A, Sharif M, Sarvi S, Aarabi M, Mizani A, et al. Toxoplasmosis in immunocompromised patients in Iran: a systematic review and meta-analysis. J Infect Dev Ctries. 2014;8(12):1503-10. doi: 10.3855/jidc.4796. [PubMed: 25500647].

3. Sharif M, Daryani A, Ebrahimnejad Z, Gholami S, Ahmadpour E, Borhani S, et al. Seroprevalence of anti-Toxoplasma IgG and IgM among individuals who were referred to medical laboratories in Mazandaran province, northern Iran. J Infect Public Health. 2016;9(1):75-80. doi:10.1016/j.jiph.2015.06.006. [PubMed: 26159578].
4. Mahami-Oskouei M, Hamidi F, Talebi M, Farhoudi M, Taheraghdam AA, Kazemi T, et al. Toxoplasmosis and Alzheimer: can Toxoplasma gondii really be introduced as a risk factor in etiology of Alzheimer? Parasitol Res. 2016;115(8):3169-74. doi: 10.1007/s00436-016-5075-5. [PubMed: 27106237].

5. Mahami Oskouei M, Hamidi F, Talebi M, Farhoudi M, Taheraghdam AA, Kazemi T, et al. The correlation between Toxoplasma gondii infection and Parkinson's disease: a case-control study. J Parasit Dis. 2016;40(3):872-6. doi: 10.1007/s12639-014-0595-3. [PubMed: 27605800].

6. Menati Rashno M, Fallahi S, Kheirandish F, Bagheri S, Kayedi MH, Birjandi M. Seroprevalence of Toxoplasma gondii infection in patients with alzheimer's disease. Arch Clin Infect Dis. 2016;11(3) doi: 10.5812/archcid.37205.

7. Sepulveda-Arias JC, Gomez-Marin JE, Bobic B, Naranjo-Galvis CA, Djurkovic-Djakovic O. Toxoplasmosis as a travel risk. Travel Med Infect Dis. 2014;12(6 Pt A):592-601. doi: 10.1016/j.tmaid.2014.05.007. [PubMed: 24951321].

8. Moncada PA, Montoya JG. Toxoplasmosis in the fetus and newborn: an update on prevalence, diagnosis and treatment. Expert Rev Anti Infect Ther. 2012;10(7):815-28. doi: 10.1586/eri.12.58. [PubMed: 22943404].

9. Jones JL, Dargelas V, Roberts J, Press C, Remington JS, Montoya JG. Risk factors for Toxoplasma gondii infection in the United States. Clin Infect Dis. 2009;49(6):878-84. doi:10.1086/605433. [PubMed: 19663709].

10. Jones JL, Parise ME, Fiore AE. Neglected parasitic infections in the United States: toxoplasmosis. Am J Trop Med Hyg. 2014;90(5):794-9. doi: 10.4269/ajtmh.13-0722. [PubMed: 24808246].

11. Derouin F, Pelloux H, Escmid Study Group on Clinical Parasitology . Prevention of toxoplasmosis in transplant patients. Clin Microbiol Infect. 2008;14(12):1089-101. doi: 10.1111/j.1469-0691.2008.02091.x. [PubMed: 19018809].

12. Elhence P, Agarwal P, Prasad KN, Chaudhary RK. Seroprevalence of Toxoplasma gondii antibodies in North Indian blood donors: implications for transfusion transmissible toxoplasmosis. Transfus Apher Sci. 2010;43(1):37-40. doi: 10.1016/j.transci.2010.05.004. [PubMed: 20605111].

13. Shaddel M, Mirzaii Dizgah I, Sharif F. The prevalence of toxoplasmosis in Imam Reza Hospital blood bank samples, Tehran, Iran. Transfus Apher Sci. 2014;51(2):181-3. doi: 10.1016/j.transci.2014.08.017. [PubMed: 25219635].

14. Hamidi F, Etemadi J, Ghabouli Mehrabani N, Mahami Oskouei M, Motavalli R, Ardalan MR. Comparison of Toxoplasma gondii seropositivity in hemodialysis and peritoneal dialysis patients. J Coast Life Med. 2015;3(8):621-2. doi: 10.12980/jclm.3.2015j5-76.

15. Mahmoudvand H, Saedi Dezaki E, Soleimani S, Baneshi MR, Kheirandish F, Ezatpour B, et al. Seroprevalence and risk factors of Toxoplasma gondii infection among healthy blood donors in southeast of Iran. Parasite Immunol. 2015;37(7):362-7. doi: 10.1111/pim.12198. [PubMed: 25891186].

16. Chiang TY, Hsieh HH, Kuo MC, Chiu KT, Lin WC, Fan CK, et al. Seroepidemiology of Toxoplasma gondii Infection among healthy blood donors in Taiwan. PLoS One. 2012;7(10):e48139. doi: 10.1371/journal.pone.0048139. [PubMed: 23133557].

17. Alvarado-Esquivel C, Mercado-Suarez MF, Rodriguez-Briones A, FalladTorres L, Ayala-Ayala JO, Nevarez-Piedra LJ, et al. Seroepidemiology of infection with Toxoplasma gondii in healthy blood donors of Durango, Mexico. BMC Infect Dis. 2007;7:75. doi: 10.1186/1471-2334-7-75. [PubMed: 17629901].

18. Alvarado-Esquivel C, Torres-Castorena A, Liesenfeld O, Garcia-Lopez CR, Estrada-Martinez S, Sifuentes-Alvarez A, et al. Seroepidemiology of Toxoplasma gondii infection in pregnant women in rural Durango, Mexico. J Parasitol. 2009;95(2):271-4. doi: 10.1645/GE-1829.1. [PubMed: 18922040].

19. Liu Q, Wang ZD, Huang SY, Zhu XQ. Diagnosis of toxoplasmosis and typing of Toxoplasma gondii. Parasit Vectors. 2015;8:292. doi: 10.1186/s13071-015-0902-6. [PubMed: 26017718]. 
20. Montoya JG. Laboratory diagnosis of Toxoplasma gondii infection and toxoplasmosis. J Infect Dis. 2002;185 Suppl 1:S73-82. doi: 10.1086/338827. [PubMed: 11865443].

21. Hill D, Dubey JP. Toxoplasma gondii: transmission, diagnosis and prevention. Clin Microbiol Infect. 2002;8(10):634-40. [PubMed: 12390281].

22. Arab-Mazar Z, Fallahi S, Koochaki A, Haghighi A, Seyyed Tabaei SJ. Immunodiagnosis and molecular validation of Toxoplasma gondiirecombinant dense granular (GRA) 7 protein for the detection of toxoplasmosis in patients with cancer. Microbiol Res. 2016;183:53-9. doi: 10.1016/j.micres.2015.11.006. [PubMed: 26805618].
23. Fallahi S, Mazar ZA, Ghasemian M, Haghighi A. Challenging loopmediated isothermal amplification (LAMP) technique for molecular detection of Toxoplasma gondii.Asian PacJTrop Med. 2015;8(5):366-72. doi: 10.1016/S1995-7645(14)60345-X. [PubMed: 26003595].

24. Prusa AR, Hayde M, Unterasinger L, Pollak A, Herkner KR, Kasper DC. Evaluation of the Roche Elecsys Toxo IgG and IgM electrochemiluminescence immunoassay for the detection of gestational Toxoplasma infection. Diagn Microbiol Infect Dis. 2010;68(4):352-7. doi: 10.1016/j.diagmicrobio.2010.07.011. [PubMed: 20884150]. 